\title{
Treatment outcome analysis of chemotherapy combined with modulated electro-hyperthermia compared with chemotherapy alone for recurrent cervical cancer, following irradiation
}

\author{
SUN YOUNG LEE ${ }^{1,2^{*}}$, NA RI LEE ${ }^{2,3^{*}}$, DONG-HYU CHO ${ }^{2,4}$ and JUNG SOO KIM ${ }^{1,2}$ \\ ${ }^{1}$ Department of Radiation Oncology, Institute for Medical Sciences, Chonbuk National University Medical School; ${ }^{2}$ Institute \\ of Clinical Medicine, Chonbuk National University-Biomedical Research Institute, Chonbuk National University Hospital; \\ ${ }^{3}$ Division of Hematology/Oncology, Department of Internal Medicine; ${ }^{4}$ Department of Obstetrics and Gynecology, \\ Chonbuk National University Hospital-Chonbuk National University Medical School, \\ Jeonju, Jeollabukdo 561-712, Republic of Korea
}

Received January 30, 2016; Accepted February 23, 2017

DOI: 10.3892/ol.2017.6117

\begin{abstract}
The survival of patients with recurrent cervical cancer following irradiation remains poor. Chemotherapy combined with hyperthermia has been demonstrated to improve the response rate. The present study was performed to evaluate the effect of modulated electro-hyperthermia combined with conventional chemotherapy compared with chemotherapy alone on recurrent cervical cancer previously treated with irradiation. A total of 20 patients were treated with chemotherapy alone, and 18 were treated with chemotherapy combined with modulated electro-hyperthermia. A single patient was treated with chemo-radiotherapy as primary treatment and then relapsed; the tumor was inoperable and radio-refractory upon recurrence. Local metastases, including metastasis of the para-aortic lymph nodes and adjacent pelvic lymph nodes were included, but distant metastases were excluded. Modulated electro-hyperthermia was performed three times per week beginning at chemotherapy initiation, and patients underwent a total of 36 sessions. The overall response (complete remission + partial remission + stable disease/progressive disease) to treatment was significantly greater in the group of patients who underwent chemotherapy combined with modulated electro-hyperthermia $(\mathrm{P}=0.0461)$, and at the evaluation conducted at the last follow-up visit, the response rate was significantly higher $(\mathrm{P}=0.0218)$. Additionally,
\end{abstract}

Correspondence to: Professor Jung Soo Kim, Department of Radiation Oncology, Institute for Medical Sciences, Chonbuk National University Medical School, 20 Geonji-ro, Jeonju, Jeollabukdo 561-712, Republic of Korea

E-mail: jskim@jbnu.ac.kr

*Contributed equally

Key words: concurrent chemo-modulated electro-hyperthermia, chemotherapy alone, recurrent cervical cancer, treatment outcome severe complications were not reported. In the present study, of patients with recurrent cervical cancer previously treated with irradiation, the overall response rate for patients treated with chemotherapy combined with modulated electro-hyperthermia was significantly greater than that for those treated with chemotherapy alone.

\section{Introduction}

Carcinoma of the uterine cervix is the fourth most common female malignancy globally, following breast, colorectal and lung cancers (1). In developed countries, including the USA, 12,900 women are diagnosed with cervical cancer and $\sim 4,100$ mortalities per year are attributed to this disease (2). The 5-year overall survival rate is $68 \%$ for all stages of cervical cancer; however, the survival rate is $91 \%$ when the disease is diagnosed in an early phase. Patients with metastases in regional lymph nodes only have a 57\% 5-year survival rate, and those with distant metastases only have a 16\% 5-year survival rate. The overall 5-year survival rate is relatively high in Korea (80-81.2\% over the last 15-year period) (3). Increased cancer screening and updated treatment strategies are key factors that have produced improved results (4). Specifically, the survival of women with recurrent cervical cancer or para-aortic lymph node (PAN) metastasis remains poor. More effective treatment methods and regimens are therefore needed. Cisplatin is an effective single therapeutic regimen for first-line therapy, but single-agent regimens for recurrent cervical cancer have a poor response rate (only $13 \%$ ) (5). The recurrence rate is lower for patients with a pelvic recurrence within previously irradiated areas than for those with extra-pelvic sites of disease. In addition, the responsiveness of pelvic recurrences is partial at best and has a brief (median 4-6 months) duration (6). To enhance the therapeutic response, chemotherapy was combined with hyperthermia (cisplatin + hyperthermia for previously radiated cases), which has been demonstrated to be feasible (7) when used in tri-modal applications for the cervix (8-11). By using local hyperthermia and systemic administration of chemotherapy, it 
is possible to achieve maximum synergism without increasing the systemic side effects, which primarily affect the bone marrow and kidneys (12). Modulated electro-hyperthermia, which was used in the present study, is a type of hyperthermia used in oncological treatments that avoids the drawbacks of conventional electromagnetic heating $(13,14)$. This treatment device is designed to selectively heat malignant tumors and tumor cells by modularly delivering $13.56 \mathrm{MHz}$ RF (15-18). This method works by heating the malignant cells, selectively and effectively acting on the cell membrane (19), and inducing apoptotic cell death (20). This advanced treatment produces damage-associated cellular patterns and promotes immunogenic cell death (21). The heat-induced increase in the tumor response to radiotherapy is due, at least in part, to an increase in the oxygen supply via increased blood circulation in tumors. The enhanced response of tumors to chemotherapy may be due to three factors. First, mild heating increases the delivery of chemotherapy drugs to the tumors by increasing blood flow to and within the tumor. Second, mild heating increases the cellular uptake of drugs by increasing cell membrane permeability. Third, heating facilitates the reaction rate of drugs, which potentiates their cytotoxicity $(21,22)$.

This method uses modulated radiofrequency for energy delivery and achieves selective thermal action in non-homogeneous tissue (23). It is also notably gentle, and its use on brain malignancies has been successful (24-26), even at increased doses for advanced cases (16). Modulated electro-hyperthermia does not cause pain and has fewer side effects, which results in improved patient satisfaction and quality of life (18). Patients treated with modulate electro-hyperthermia have reported pain relief (23) and, as a result, a decreased dose of analgesic is required (22). The aim of the present study was to evaluate the effect of modulated electro-hyperthermia combined with conventional chemotherapy compared with chemotherapy alone on recurrent cervical cancer previously treated with irradiation, by analyzing the outcomes of patients subjected to each treatment. The objectives of the present study were to determine the local control, treatment outcome and side effects.

\section{Materials and methods}

Ethics statement. The present study was approved by the Institutional Review Board of Chonbuk National University Hospital (Jeonju, Republic of Korea) and was conducted according to the Declaration of Helsinki regarding biomedical research involving human subjects and the Guidelines for Good Clinical Practice. Written, informed consent was obtained from all patients.

Patients. A total of 20 subjects were included in the chemotherapy only group, and 18 subjects were included in the chemotherapy combined with modulated electro-hyperthermia group and all patients underwent treatment at the Chonbuk National University Hospital (Table I). Patients were included in the present study if they had histologically confirmed cervical cancer (histological diagnosis of squamous cell carcinoma or adenocarcinoma) and if complete remission (CR) was achieved following first-line treatment. In addition, a patient who experienced recurrence following surgery and chemo-radiotherapy was included in the present study. A patient who received chemo-radiotherapy as primary treatment suffered a relapse; the tumor was inoperable and radio-refractory following recurrence. Local metastases were included, for example metastasis of the PANs or the adjacent pelvic lymph nodes (PLNs), but distant metastases were excluded. The selected patients were required to have an ECOG status of $\leq 3$. Patients underwent 36 modulated electro-hyperthermia sessions and at least 6 months of follow-up. Patients in the chemotherapy only group had the following indications: 1 , recurrence in a PAN alone or in a PLN and/or the bed of the cervix; 2, no distant metastasis (excluding PANs); 3, radiotherapy was not possible due to recurrence in a previous radiotherapy field; and 4, at least 6 months of follow-up.

Patients in the chemotherapy combined with modulated electro-hyperthermia group had the following indications: i) Recurrence in a PAN or PLN and the bed of the cervix (specifically, in the $30 \mathrm{~cm}$ probe treatment area); ii) no distant metastasis (excluding PANs); iii) 36 sessions of hyperthermia therapy (3 sessions per week); iv) radiotherapy was not possible due to recurrence in a previous radiotherapy field; and v) at least 6 months of follow-up.

Chemotherapy. All patients received platinum-based chemotherapy for recurrence according to local hospital policy. The treatment characteristics are summarized in Table II. Depending on local hospital policy, the goal of combined chemotherapy with modulated electro-hyperthermia was to administer 36 sessions of modulated electro-hyperthermia during chemotherapy. All patients were evaluated for toxicity each week.

Modulated electro-hyperthermia. Modulated electro-hyperthermia treatment was applied using an EHY2000 clinical heating device (Oncotherm $\mathrm{GmbH}$, Troisdorf, Germany) set at a $13.56 \mathrm{MHz}$ carrier frequency, and the amplitude was modulated according to a time fractal pattern. Modulated electro-hyperthermia was performed for $60 \mathrm{~min}$. The patients were placed in a supine position on a water mattress electrode. A circular upper electrode (30 cm in diameter) was coupled over the recurrent cervical mass or PAN mass. Prior to modulated-electro hyperthermia, all patients underwent a two-dimensional simulation. The treatment field encompassed the mass with a $3 \mathrm{~cm}$ margin in the $\mathrm{X}, \mathrm{Y}$, and $\mathrm{Z}$ directions. Modulated electro-hyperthermia was performed three times each week beginning at the initiation of chemotherapy, and patients underwent a total of 36 sessions.

The power output was $80 \mathrm{~W}$ for the first $10 \mathrm{~min}, 120 \mathrm{~W}$ for the next $10 \mathrm{~min}$ and $150 \mathrm{~W}$ for the remaining treatment time. Self-calibration of the device was performed prior to each treatment (11). The body temperature, blood pressure and pulse rate of each patient were measured prior to, during and following treatment. Body temperature was measured using an infrared ear thermometer (Infrared Thermometer IRT 4020; Braun $\mathrm{GmbH}$, Kronberg, Germany) and the temperature of the abdominal skin surface below the circular upper electrode probe was measured using a non-contact infrared thermometer transmitter (Thermo Checker DT-060; Easytem Co., Ltd., Siheung, Korea). Adverse events (AEs) of the associated-hyperthermia and chemotherapy were monitored 
Table I. Characteristics of patients with recurrent cervical cancer who had previously undergone chemo-radiotherapy, who were subsequently subjected to chemotherapy alone or chemotherapy combined with modulated electro-hyperthermia.

\begin{tabular}{lcc}
\hline Parameter & Chemotherapy alone $(\mathrm{n}=20)$ & Chemotherapy and modulated electro-hyperthermia $(\mathrm{n}=18)$ \\
\hline Age, years & $36-71$ & $36-71$ \\
Range & 53 & 50.8 \\
Mean & & 3 \\
FIGO stage & 2 & 3 \\
Ib & 3 & 3 \\
IIa & 3 & 2 \\
IIb & 5 & 4 \\
IIIa & 4 & 3 \\
IIIb & 3 & 15 \\
IVa & & 3 \\
Pathology & 15 & \\
Squamous cell carcinoma & 5 & 6 \\
Adenocarcinoma & & 4 \\
Recurrent lesions & 4 & 5 \\
Cervix bed alone & 4 & 3 \\
PAN alone & 4 &
\end{tabular}

PAN, para-aortic lymph node; LN, lymph node.

Table II. Treatment characteristics of the chemotherapy alone and chemotherapy combined with modulated electro-hyperthermia groups.

\begin{tabular}{lcccc}
\hline Group & TP (cycle) & TC (cycle) & FP (cycle) & Cisplatin (cycle) \\
\hline Chemotherapy alone $(\mathrm{n}=20)$ & $8(5-7)$ & $6(6-9)$ & $6(4-6)$ & 0 \\
Chemotherapy and modulated electro-hyperthermia $(\mathrm{n}=18)$ & $6(5-6)$ & $4(6)$ & $6(4-6)$ & $2(5-6)$ \\
\hline
\end{tabular}

TP, paclitaxel+cisplatin; TC, paclitaxel+carboplatin; FP, cisplatin+5-fluorouracil.

Table III. Clinical response of the chemotherapy-alone and the chemotherapy-combined with modulated electro-hyperthermia groups following completion of treatment.

\begin{tabular}{|c|c|c|c|c|c|}
\hline Group & Complete remission & Partial response & Stable disease & Progressive disease & P-value \\
\hline Chemotherapy alone $(n=20)$ & 4 & 3 & 1 & 12 & 0.0461 \\
\hline $\begin{array}{l}\text { Chemotherapy and modulated } \\
\text { electro-hyperthermia }(n=18)\end{array}$ & 9 & 2 & 2 & 5 & \\
\hline
\end{tabular}

throughout the present study. Hyperthermia-associated adverse events were hot sensation, skin burn and gastric discomfort. Chemotherapy-associated adverse events were pancytopenia, nausea, vomiting, anorexia and gastric discomfort. AEs were determined by investigator inquiry and by spontaneous patient reports. The AEs were recorded with regard to the symptoms, signs, duration and severity (mild, moderate, and severe). Clinical safety parameters, including blood glucose levels, vital signs, 12-lead ECG results and clinical laboratory tests, were observed during the regular chemotherapy cycles (3-4 weeks intervals).

Statistics. The end points of the present study were tumor response [CR, partial remission (PR), stable disease (SD) and 
Table IV. Analysis of the last follow-up treatment response to chemotherapy alone or chemotherapy combined with modulated electro-hyperthermia.

\begin{tabular}{|c|c|c|c|c|c|}
\hline Group & Complete remission & Partial response & Stable disease & Progressive disease & P-value \\
\hline Chemotherapy alone $(n=20)$ & 3 & 3 & 1 & 13 & 0.0218 \\
\hline $\begin{array}{l}\text { Chemotherapy and modulated } \\
\text { electro-hyperthermia }(n=18)\end{array}$ & 9 & 2 & 2 & 5 & \\
\hline
\end{tabular}

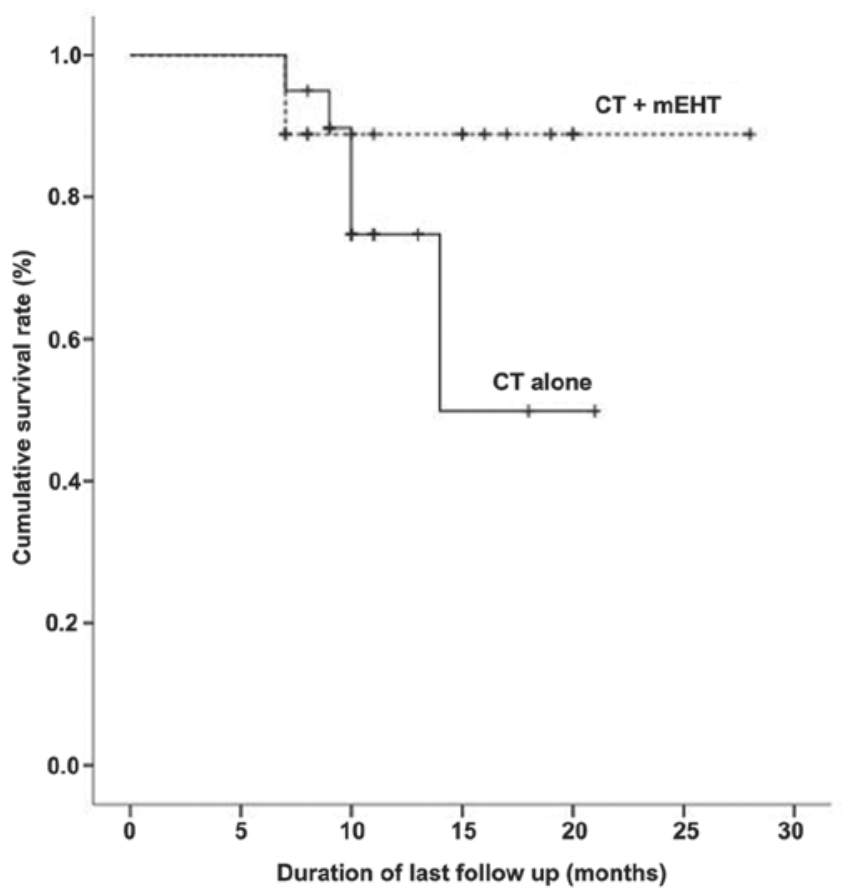

Figure 1. Overall survival of the patients treated with chemotherapy alone compared with those subjected to CT combined with mEHT groups. CT combined with mEHT did not significantly increase the overall survival rate $(\mathrm{P}=0.235)$. CT, chemotherapy; mEHT, modulated electro-hyperthermia.

progressive disease (PD)], overall survival, final follow-up status and toxicity. Student's t-test was used for treatment response analysis.

The time to an event variable was estimated using Kaplan-Meier analysis. The statistical analysis was performed using SAS software (version 9.3; SAS Institute, Inc., Cary, NC, USA). $\mathrm{P}<0.05$ was considered to indicate statistically significant difference.

\section{Results}

The 38 included patients were evaluated for treatment response. The response rates for the chemotherapy alone group $(n=20)$ and the chemotherapy combined with modulated electro-hyperthermia $(n=18)$ are summarized in Table III. For patients diagnosed with cervical cancer, the time to relapse following curative treatment was 8.95 months following the first treatment for the chemotherapy only group and 9.16 months for the combined chemotherapy with modulated electro-hyperthermia group. The overall response rate $(\mathrm{CR}+\mathrm{PR}+\mathrm{SD} / \mathrm{PD})$ to treatment was significantly greater in the group that underwent chemotherapy combined with modulated electro-hyperthermia ( $\mathrm{P}=0.0461$; Table III).

At the evaluation conducted at the final follow-up visit, the reaction was significantly greater $(\mathrm{P}=0.0218$; Table IV) in the group that underwent chemotherapy combined with modulated electro-hyperthermia compared with the group that underwent chemotherapy alone.

In the case of chemotherapy alone, statistically significant recurrence was only observed when the cervix was the target of the therapeutic response $(\mathrm{P}=0.0456)$, but in the chemotherapy combined with modulated electro-hyperthermia, no significant differences between abdominal lymph node and cervical recurrences were observed $(\mathrm{P}=0.6199)$. The follow-up period was 7-21 months (mean 11 months) for the chemotherapy only group and the follow-up period was 7-28 months (mean 13.5 months) for the chemotherapy combined with modulated electro-hyperthermia group. The Kaplan-Meier plot of overall survival is presented in Fig. 1. Based on the overall survival rates of the two groups, chemotherapy combined with modulated electro-hyperthermia did not significantly increase disease-free survival compared with chemotherapy alone $(\mathrm{P}=0.235)$.

The body temperature ranged from $36.4-36.9^{\circ} \mathrm{C}$ (mean, $36.5^{\circ} \mathrm{C}$ ) prior to treatment and $36.9-38.2^{\circ} \mathrm{C}$ (mean, $37.6^{\circ} \mathrm{C}$ ) following modulated electro-hyperthermia indicating an average increase of $1.1^{\circ} \mathrm{C}$. The temperature of the abdominal skin surface below the upper electrode ranged from $36.4-37.3^{\circ} \mathrm{C}$ (mean, $36.8^{\circ} \mathrm{C}$ ) prior to heating, and increased to $39.8-41.9^{\circ} \mathrm{C}$ (mean, $40.8^{\circ} \mathrm{C}$ ), for an average surface temperature increase of $\sim 4^{\circ} \mathrm{C}$ (Fig. 2).

The majority of patients suffered the following chemotherapy complications: Nausea, vomiting, pancytopenia and peripheral neuropathy. However, severe chemotherapy-related complications did not occur. The side effects included hot sensations and abdominal discomfort for 8/18 (44\%) patients, and no other side effects (burns or blisters) were reported. No late toxicity associated with modulated electro-hyperthermia was observed.

The pathological characteristics, chemotherapeutic regimens, cycles, dosages and ages were not significantly different with respect to treatment response.

\section{Discussion}

Surgery, chemotherapy, radiotherapy, or a combination of these modalities continues to be the primary treatment option for invasive cervical cancer. However, following surgery and postoperative radiotherapy, primary definitive concurrent chemo-radiation therapy or radiotherapy alone results in 


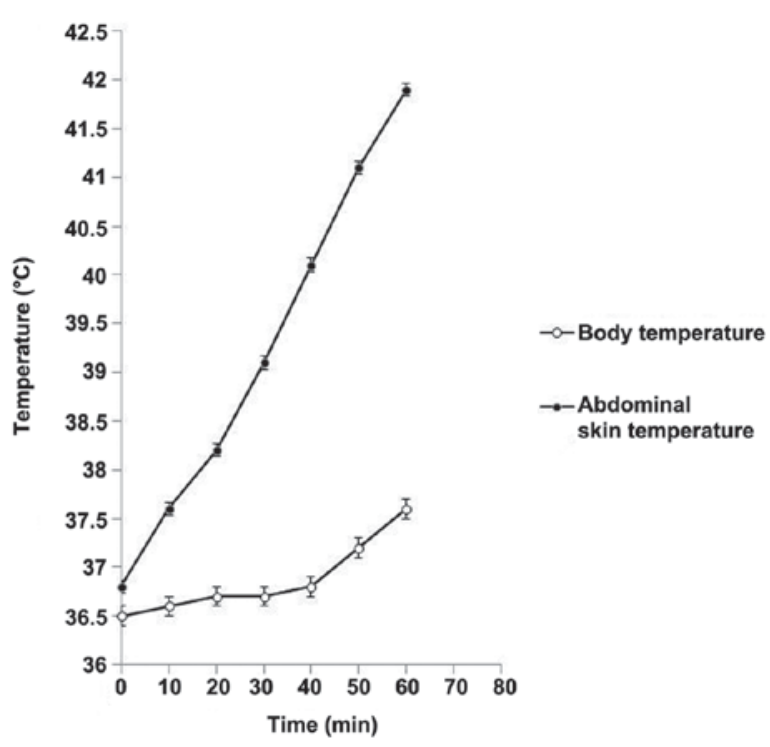

Figure 2. Body temperature and abdominal skin surface temperature underneath the electrode, during modulated electro-hyperthermia.

the development of recurrent pelvic disease in up to $40 \%$ of patients. With the exception of salvage surgery as a treatment option for certain individuals among these patients, the use of systemic chemotherapy is the only remaining treatment modality (27). Cisplatin has emerged as the most active single agent for treating patients with metastatic disease, and no other standard cytotoxic drug has been consistently associated with objective response rates of $\geq 25 \%$. However, for patients who experience relapse following definitive radiation therapy, cisplatin has only a minor effect $(6,28)$.

In a study by Potter et al (29), the complete response rate to cisplatin in patients with distant metastases was $53 \%$ with an overall response rate of $73 \%$, whereas no complete responses and only 7 partial responses $(21 \%)$ were observed among 33 patients with localized pelvic recurrence or persistent disease. Thus, the application of deep local hyperthermia along with the systemic administration of cisplatin appears to be an attractive option in patients with recurrent pelvic cervical cancer. Once initial feasibility data regarding the clinical use of cisplatin and local hyperthermia combination treatment were reported (29), Rietbroek et al (7) published a phase II study of combined weekly locoregional hyperthermia and systemic cisplatin administration in patients with previously irradiated recurrent cervical carcinoma (30). Using a regimen of $50 \mathrm{mg}$ of cisplatin per week with a one-week interruption following four cycles for a total of 12 cycles, corresponding to a projected dose intensity of $40 \mathrm{mg}$ per week, these authors observed an overall response in $12 / 23$ patients $(52 \%$; $95 \%$ confidence interval, 31-73\%) (7).

The expectation is that a hyperthermia-induced increase in temperature may increase blood flow in the targeted region as a homeostatic response $(31,32)$. An elevated local temperature $\left(\leq 40^{\circ} \mathrm{C}\right)$ has been demonstrated to initiate various physical and chemical processes and to ease pain in patients with cancer (27). Drug potentiation in target tissues increases the treatment response. The temperature increase induced by hyperthermia enhances the reaction rate of a given drug independent of an increase in drug delivery due to enhanced blood flow, and increases the reaction rate in target tumor cells, thereby escalating tumor cell death $(18,23)$. The thermally increased metabolism (the so-called enhanced chemo-metabolism) associated with hyperthermia also increases the absorption of cytotoxins (27) and is expected to enhance drug absorption.

It is well known that heating tumors to mild temperatures, for example $40-43^{\circ} \mathrm{C}$, kills tumor cells and increases the tumor response of radiotherapy and chemotherapy with various anti-cancer drugs $(33,34)$. The heat-induced increase in the tumor response to radiotherapy is due, at least in part, to an increase in oxygen supply via the heat-induced enhancement of intratumoral blood circulation. The heat-induced increase in the tumor response to chemotherapy may be due to the following three factors. First, mild heating increases the delivery of chemotherapy drugs to tumors by increasing tumor blood flow. Second, mild heating elevates the cellular uptake of drugs by increasing cell membrane permeability. Third, heating facilitates the reaction rate of drugs, which potentiates their cytotoxicity (27). It must be noted that the systemic body temperature may increase when tumors are treated with regional or local hyperthermia (22). It is therefore likely that such an increase in body temperature may alter the pharmacokinetics of chemotherapy drugs when chemotherapy and regional hyperthermia are concomitantly applied (35).

In the present study, concerning cervical cancer recurrence in patients previously treated with irradiation, chemotherapy combined with modulated electro-hyperthermia significantly increased the treatment response. The overall response rate $(\mathrm{CR}+\mathrm{PR}+\mathrm{SD} / \mathrm{PD})$ to treatment was significantly improved among the patients who underwent chemotherapy combined with modulated electro-hyperthermia compared with those that underwent chemotherapy alone $(\mathrm{P}=0.0461)$. At the evaluation conducted at the last follow-up visit, the results were significantly improved in the group that underwent chemotherapy combined with modulated electro-hyperthermia compared with chemotherapy alone $(\mathrm{P}=0.0218)$. According to the present study, anti-cancer treatment and hyperthermia may have a synergistic effect.

Specifically, in the case of chemotherapy alone, significant recurrence was observed only when the cervix was the target of the therapeutic response $(\mathrm{P}=0.0456)$, but in the chemotherapy combined with modulated electro-hyperthermia group, no significant differences in abdominal lymph node and cervical recurrence were observed $(\mathrm{P}=0.6199)$. Hyperthermia may therefore be slightly more effective for the treatment of abdominal lymph node metastasis.

The present study demonstrated the feasibility and advantages of chemotherapy combined with hyperthermia, concurrent with the application of platinum derivatives, for patients with recurrent cervical cancer who have regional lymph node metastasis. A longer-term follow-up study is required to compare the disease-free survival rates of patients.

\section{Acknowledgements}

The present study was supported by funds from the Institute of Clinical Medicine of Chonbuk National University-Biomedical Research Institute, Chonbuk National University Hospital. 


\section{References}

1. International Agency for Research on Cancer: World Health Organization. Latest world cancer statistics. IARC, Lyon, 2013.

2. American Society of Clinical Oncology (ASCO): Cervical cancer: Statistics. Available at http://www.cancer.net/cancer-types/ cervical-cancer/statistics.

3. Korean statistical information service. Statistics Korea. Statistics Korea, Daejeon, 2012. Available from: http://kosis.kr.

4. Seol HJ, Ki KD and Lee JM: Epidemiologic characteristics of cervical cancer in Korean women. J Gynecol Oncol 25: 70-74, 2014.

5. Kuo DY, Blank SV, Christos PJ, Kim M, Caputo TA, Pothuri B, Hershman D, Goldman N, Ivy PS, Runowicz CD, et al: Paclitaxel plus oxaliplatin for recurrent or metastatic cervical cancer: A New York cancer consortium study. Gynecol Oncol 116 : 442-446. 2010

6. de Wit R, van der Zee J, van der Burg ME, Kruit WH, Logmans A, van Rhoon GC and Verweij J: A phase I/II study of combined weekly systemic cisplatin and locoregional hyperthermia in patients with previously irradiated recurrent carcinoma of the uterine cervix. Br J Cancer 80: 1387-1391, 1999.

7. Rietbroek RC, Schilthuis MS, Bakker PJ, van Dijk JD, Postma AJ, González González D, Bakker AJ, van der Velden J, Helmerhorst TJ and Veenhof CH: Phase II trial of weekly locoregional hyperthermia and cisplatin in patients with a previously irradiated recurrent carcinoma of the uterine cervix. Cancer 79: 935-943, 1997.

8. Jones EL, Samulski TV, Dewhirst MW, Alvarez-Secord A Berchuck A, Clarke-Pearson D, Havrilesky LJ, Soper J and Prosnitz LR: A pilot phase II trial of concurrent radiotherapy, chemotherapy, and hyperthermia for locally advanced cervical carcinoma. Cancer 98: 277-282, 2003

9. Tsuda $\mathrm{H}$, Tanaka M, Manabe T, Ikeda $\mathrm{H}$, Negoro S, Ishiko $\mathrm{O}$ and Yamamoto K: Phase I study of combined radiation, hyperthermia and intra-arterial carboplatin for local recurrence of cervical cancer. Ann Oncol 14: 298-303, 2003.

10. Westermann AM, Jones EL, Schem BC, van der Steen-Banasik EM, Koper P, Mella O, Uitterhoeve AL, de Wit R, van der Velden J, Burger C, et al: First results of triple-modality treatment combining radiotherapy, chemotherapy, and hyperthermia for the treatment of patients with stage IIB, III, and IVA cervical carcinoma. Cancer 104: 763-770, 2005

11. Franckena M, De Wit R, Ansink AC, Notenboom A, Canters RA, Fatehi D, Van Rhoon GC and Van Der Zee J: Weekly systemic cisplatin plus locoregional hyperthermia: An effective treatment for patients with recurrent cervical carcinoma in a previously irradiated area. Int J Hyperthermia 23: 443-450, 2007.

12. Dahl O: Interaction of heat and drugs in vitro and in vivo. In: Thermoradiotherapy and Thermochemotherapy. Seegenschmiedt MH, Fessenden P and Vernon CC (eds). Vol. 1. Springer Verlag, Berlin, pp103-121, 1995.

13. Hegyi G, Szigeti GP and Szász A: Hyperthermia versus oncothermia: Cellular effects in complementary cancer therapy. Evid Based Complement Alternat Med 2013: 672873, 2013.

14. Andocs G, Okamoto Y, Kawamoto K, Osaki T, Tsuka T, Imagawa T, Minami S, Balogh L, Meggyeshazi N and Szasz O: Oncothermia basic research at in vivo level: The first results in Japan. Conference Papers Med 2013: 197328, 2013.

15. Szasz A, Vincze G, Szasz O and Szasz N: An energy analysis of extracellular hyperthermia. Electromagn Biol Med 22: 103-115, 2003.

16. Meggyeshazi N, Andocs G, Balogh L, Balla P, Kiszner G, Teleki I, Jeney A and Krenacs T: DNA fragmentation and caspase-independent programmed cell death by modulated electrohyperthermia. Strahlenther Onkol 190: 815-822, 2014

17. Andocs G, Meggyeshazi N, Balogh L, Spisak S, Maros ME, Balla P, Kiszner G, Teleki I, Kovago C and Krenacs T: Upregulation of heat shock proteins and the promotion of damage associated molecular pattern signals in a colorectal cancer mode by modulated electro-hyperthermia. Cell Stress Chaperones 20 $37-46,2014$
18. Szasz A: Challenges and solutions in oncological hyperthermia Med 29: 1-23, 2013.

19. Fiorentini G, Giovanis P, Rossi S, Dentico P, Paola R, Turrisi G and Bernardeschi P: A phase II clinical study on relapsed malignant gliomas treated with electro-hyperthermia. In Vivo 20: 721-724, 2006.

20. Hager ED, Sahinbas H, Groenemeyer DH and Migeod F: Prospective phase II trial for recurrent high-grade malignant gliomas with capacitive coupled low radiofrequency (LRF) deep hyperthermia. J Clin Oncol 26: 2047, 2008.

21. Sahinbas H, Grönemeyer DH, Böcher E and Szasz A: Retrospective clinical study of adjuvant electro-hyperthermia treatment for advanced brain-gliomas. Dtsch Z Onkol 39: 154-160, 2007.

22. Wismeth C, Dudel C, Pascher C, Ramm P, Pietsch T, Hirschmann B, Reinert C, Proescholdt M, Rümmele P, Schuierer G, et al: Transcranial electro-hyperthermia combined with alkylating chemotherapy in patients with relapsed high-grade gliomas: Phase I clinical results. J Neurooncol 98: 395-405, 2010.

23. Andocs G, Szasz O and Szasz A: Oncothermia treatment of cancer: From the laboratory to clinic. Electromagn Biol Med 28: $148-165,2009$

24. Szasz A: Current status of oncothermia therapy for Lung cancer. Korean J Thorac Cardiovasc Surg 47: 77-93, 2014.

25. Jeung TS, Ma SY, Yu J and Lim S: Cases that respond to Oncothermia Monotherapy. Conference Papers Med 2013: 392480, 2013

26. Szasz A, Iluri N and Szasz O: Local hyperthermia in oncologyto choose or not to choose? In: Hyperthermia. Huilgol N (eds). InTech, Croatia, pp1-82, 2013.

27. Gonzalez-Gonzalez D: Thermo-radiotherapy for tumors of the lower gastro-instenstinal tract. In: Thermoradiotherapy and thermochemiotherapy: Biology, physiology, physics. Seegenschmiedt MH. Fessenden P and Vernon CC (eds). Springer Verlag, Berlin, pp105-109, 1996.

28. Brader KR, Morris M, Levenback C, Levy L, Lucas KR and Gershenson DM: Chemotherapy for cervical carcinoma: Factors determining response and implications for clinical trial design. J Clin Oncol 16: 1879-1884, 1998.

29. Potter ME, Hatch KD, Potter MY, Shingleton HM and Baker VV: Factors affecting the response of recurrent squamous cell carcinoma of the cervix to cisplatin. Cancer 63: 1283-1286, 1989.

30. Green DM, Burton GV, Cox EB, Hanson D, Moore J and Oleson JR: A phase I/II study of combined cisplatin and hyperthermia treatment for refractory malignancy. Int J Hyperthermia 5: 13-21, 1989.

31. Song CW: Effect of local hyperthermia on blood flow and microenvironment: A review. Cancer Res 44 (10 Suppl): 4721s-4730s, 1984.

32. Hegyi G, Vincze G and Szasz A: On the dynamic equilibrium in homeostasis. Open J Biophys 2: 64-71, 2012.

33. Urano M, Kuroda M and Nishimura Y: For the clinical application of thermochemotherapy given at mild temperatures. Int J Hyperthermia 15: 79-107, 1999.

34. Urano M, Wong KH, Reynolds R and Begley J: The advantageous use of hypoxic tumour cells in cancer therapy: Identical chemosensitization by metronidazole and misonidazole at moderately elevated temperatures. Int J Hyperthermia 11: 379-388, 1995.

35. Heijkoop ST, van Doorn HC, Stalpers LJ, Boere IA, van der Velden J, Franckena $M$ and Westermann AM: Results of concurrent chemotherapy and hyperthermia in patients with recurrent cervical cancer after previous chemoradiation. Int J Hyperthermia 30: 6-10, 2014. 